OU-HET 587/2007

KOBE-TH-07-08

\title{
A Simple Model of Direct Gauge Mediation of Metastable Supersymmetry Breaking
}

\author{
Naoyuki Haba*1 and Nobuhito Maru坥 \\ ${ }^{*}$ Department of Physics, Osaka University, Toyonaka, Osaka 560-0043, Japan \\ ${ }^{\dagger}$ Department of Physics, Kobe University, Kobe 657-8501, Japan
}

\begin{abstract}
We construct a model of direct gauge mediation of metastable SUSY breaking by simply deforming the Intriligator, Seiberg and Shih model in terms of a dual meson superpotential mass term. No extra matter field is introduced. The deformation explicitly breaks a $U(1)_{R}$ symmetry and a pseudo moduli have a nonzero VEV at one-loop. Our metastable SUSY breaking vacuum turns out to be sufficiently long-lived. By gauging a subgroup of flavor symmetry, our model can directly couple to the standard model, which leads to nonvanishing gaugino mass generation. It is also shown that our model can evade the Landau pole problem. We show the parameters in the SUSY breaking sector are phenomenologically constrained.
\end{abstract}

\footnotetext{
${ }^{1}$ e-mail: haba@het.phys.sci.osaka-u.ac.jp

${ }^{2}$ e-mail: maru@people.kobe-u.ac.jp
} 


\section{Introduction}

Dynamical supersymmetry (SUSY) breaking is a convincing scenario to solve the gauge hierarchy problem [1]. According to Witten index [2], the models of dynamical SUSY breaking were limited to the chiral models since the vector-like models except for the models discussed in [3, 4] do not break SUSY similar to super Yang-Mills theories. Building such chiral models with dynamical SUSY breaking was very troublesome. Once one can obtain SUSY breaking models, the next issue is how to transmit the SUSY breaking to our real world. The gauge mediation where the SUSY breaking effects are transmitted to our world by the Standard Model(SM) gauge interactions is one of the attractive framework since the gauge interaction is flavor-blind, thus there is no SUSY FCNC problem [5, 6]. If we try to communicate the SUSY breaking directly to our world by gauging a subgroup of the flavor symmetry in the chiral SUSY breaking models known before and identifying it with the SM gauge group, it is likely that the QCD coupling blows up below the GUT scale [7]. This is because the number of color is always larger than the flavor number in such chiral SUSY breaking models, which leads to the large number of messengers. This led to the introduction of the messenger sector [6], but the sector was in general quite complicated. Some models of direct gauge mediation avoiding the Landau pole problem were constructed so far [8]-[14].

Recently, Intriligator, Seiberg and Shih (ISS) have discovered a metastable SUSY breaking vacuum in $\mathcal{N}=1$ massive (but light) SUSY QCD in a free magnetic phase [15]. The typical model is an $\mathcal{N}=1 \mathrm{SUSY} S U\left(N_{c}\right)$ gauge theory with massive (but light) $N_{f}$ quark chiral multiplets in the range $N_{c}<N_{f}<\frac{3}{2} N_{c}$ [16]. In spite that the SUSY breaking vacuum is metastable, their finding is quite remarkable in that it opened a new avenue to overcome the two major difficulties mentioned in the previous paragraph. First, the model building becomes very easy since the ISS models are vector-like. Second, we can guess that the Landau pole problem is relaxed since the ISS models has a large flavor number comparing to the number of color, which reduces the number of messengers. Motivated by the proposal of ISS, extensive researches on the metastable SUSY breaking have been carried out in various viewpoints [17]-[36].

There is, however, a serious problem in the ISS model. $U(1)_{R}$ symmetry is unbroken at the metastable SUSY breaking vacuum, which implies that the gauginos cannot obtain a Majorana mass. How the $U(1)_{R}$ is broken is the first step to the model building of metastable SUSY breaking. Some models of direct mediation of metastable SUSY breaking with broken $U(1)_{R}$ symmetry have been proposed using the inverted hierarchy mechanism [20, 24] and the explicit $U(1)_{R}$ breaking superpotential term [21, 34].

In this paper, we construct a direct gauge mediation model of metastable SUSY break- 
ing by simply deforming the ISS model in terms of a dual meson superpotential mass term. No extra matter is introduced. The deformation explicitly breaks $U(1)_{R}$ symmetry. We show that the vacuum expectation value (VEV) of the pseudo moduli is located at the nonzero value and messenger fields obtain SUSY masses through the Coleman-Weinberg potential. Our metastable SUSY breaking vacuum turns out to be sufficiently long-lived. Gauging a subgroup of the flavor symmetry and identifying it with the SM gauge group, the direct gauge mediation is realized and nonvanishing gaugino masses are generated. We also find that Landau pole problem can be avoided. These analyses show the parameters in the dynamical SUSY breaking sector are phenomenologically constrained.

\section{Model}

In this section, we consider a metastable SUSY breaking model of ISS deformed by a dual meson superpotential mass term which breaks a $U(1)_{R}$ explicitly. The model is an $\mathcal{N}=1$ SUSY $S U\left(N_{f}-N_{c}\right)$ gauge theory with $N_{f}$ flavor dual quarks $q, \tilde{q}$ and the gauge singlet $M$ couples to the dual quarks in the superpotential, which is a free magnetic description of SUSY $S U\left(N_{c}\right)$ gauge theory with massive $N_{f}$ flavors. The superpotential and Kähler potential are given by

$$
\begin{aligned}
W & =\operatorname{Tr}(q M \tilde{q})+m^{2} \operatorname{Tr} M+\frac{\epsilon}{\Lambda} \operatorname{Tr}(q \tilde{q})^{2}, \\
K & =M^{\dagger} M+q^{\dagger} q+\tilde{q} \tilde{q}^{\dagger},
\end{aligned}
$$

where $m$ and $\Lambda$ are dimensionful parameters and $\epsilon$ is a dimensionless parameter. The ISS model is just deformed by the last term in the superpotential (1), which explicitly breaks the $U(1)_{R}$ symmetry. Note that the simplest deformation by the term $\operatorname{Tr} q \tilde{q}$ does not change the physics in the ISS model, namely the $U(1)_{R}$ is unbroken even at one loop level since this term can be absorbed into the first term in (1) by the constant shift of $M$.

Parametrizing $M, q, \tilde{q}$ and $m$ as,

$$
M=\left(\begin{array}{cc}
Y & Z \\
\tilde{Z} & \Phi
\end{array}\right), \quad q=\left(\begin{array}{c}
\chi \\
\rho
\end{array}\right), \quad \tilde{q}=\left(\begin{array}{c}
\tilde{\chi} \\
\tilde{\rho}
\end{array}\right), \quad m=\left(\begin{array}{cc}
m \delta_{a b} & 0 \\
0 & \tilde{m} \delta_{A B}
\end{array}\right)
$$

where $a, b=1, \cdots, N_{f}-N_{c}$ and $A, B=1, \cdots, N_{c}$ and we assumed $m$ to be in the block diagonal form. The superpotential and Kähler potential are decomposed as

$$
\begin{aligned}
W= & \operatorname{Tr}\left[\chi Y \tilde{\chi}+\chi Z \tilde{\rho}+\tilde{\chi} \tilde{Z} \rho+\rho \Phi \tilde{\rho}+m^{2} Y+\tilde{m}^{2} \Phi\right] \\
& +\frac{\epsilon}{\Lambda} \operatorname{Tr}\left[(\chi \tilde{\chi})^{2}+(\chi \tilde{\rho})(\tilde{\chi} \rho)+(\chi \tilde{\chi})(\chi \tilde{\rho})+(\chi \tilde{\rho})(\rho \tilde{\rho})+(\rho \tilde{\chi})(\chi \tilde{\chi})\right. \\
& \left.+(\rho \tilde{\rho})(\rho \tilde{\chi})+(\rho \tilde{\chi})(\chi \tilde{\rho})+(\rho \tilde{\rho})^{2}\right] \\
K= & |Y|^{2}+|Z|^{2}+|\tilde{Z}|^{2}+|\Phi|^{2}+|\chi|^{2}+|\rho|^{2}+|\tilde{\chi}|^{2}+|\tilde{\rho}|^{2} .
\end{aligned}
$$


Let us study the vacuum structure at the classical level. Using the degree of freedom of the symmetries $S U(N) \times S U\left(N_{f}\right) \times S U\left(N_{f}\right)$, we can take the VEVs of the matter fields in the following block diagonal form, namely $Z=\tilde{Z}=\rho=\tilde{\rho}=0$, and

$$
M=\left(\begin{array}{cc}
Y_{a b} \delta_{a b} & 0 \\
0 & \Phi_{A B} \delta_{A B}
\end{array}\right), \quad q=\left(\begin{array}{c}
\chi_{a b} \delta_{a b} \\
0
\end{array}\right), \quad \tilde{q}=\left(\begin{array}{c}
\tilde{\chi}_{a b} \delta_{a b} \\
0
\end{array}\right) .
$$

Note that the gauge symmetry $S U\left(N_{f}-N_{c}\right)$ is completely broken in this vacuum. Putting these VEVs into F-flatness conditions leads to

$$
\begin{aligned}
& 0=\frac{\partial W}{\partial Y}=\chi \tilde{\chi}+m^{2}, \\
& 0 \neq \frac{\partial W}{\partial \Phi}=\tilde{m}^{2}, \\
& 0=\frac{\partial W}{\partial \chi}=\left[Y+\frac{2 \epsilon}{\Lambda}(\chi \tilde{\chi})\right] \tilde{\chi}, \\
& 0=\frac{\partial W}{\partial \rho}=\frac{\epsilon}{\Lambda} \tilde{\chi}(\chi \tilde{\chi}), \\
& 0=\frac{\partial W}{\partial \tilde{\chi}}=\left[Y+\frac{2 \epsilon}{\Lambda}(\chi \tilde{\chi})\right] \chi, \\
& 0=\frac{\partial W}{\partial \tilde{\rho}}=\frac{\epsilon}{\Lambda} \chi(\chi \tilde{\chi}) .
\end{aligned}
$$

The second equation $F_{\Phi} \neq 0$ shows that SUSY is spontaneously broken. Its fermionic component $\psi_{\Phi}$ is a Nambu-Goldstone fermion and eaten by the gravitino when the gravitational coupling is switched on.

The scalar potential at tree level is given by

$$
V_{\text {tree }}=\left|\chi \tilde{\chi}+m^{2}\right|^{2}+\left|\tilde{m}^{2}\right|^{2}+\left[\left|Y+\frac{2 \epsilon}{\Lambda} \chi \tilde{\chi}\right|^{2}+\left|\frac{\epsilon}{\Lambda}(\chi \tilde{\chi})\right|^{2}\right]\left(|\chi|^{2}+|\tilde{\chi}|^{2}\right) .
$$

This potential has a local minimum with

$$
Y+\frac{2 \epsilon}{\Lambda} \chi \tilde{\chi}=0
$$

and a local maximum with

$$
|\chi|=|\tilde{\chi}|=0, Y \text { (arbitrary) }
$$

where $Y$ is undetermined at tree level. From now, we focus on the case (14). The minimization conditions in this case is given by

$$
0=\chi \tilde{\chi}+m^{2}+2\left(\frac{\epsilon}{\Lambda}\right)^{2} \chi \tilde{\chi}|\chi|^{2}+\left|\frac{\epsilon}{\Lambda} \chi \tilde{\chi}\right|^{2}
$$

where D-flat condition $|\chi|=|\tilde{\chi}|$ is used. Expanding VEVs as

$$
\begin{aligned}
\chi \tilde{\chi} & =-m^{2}+\epsilon(\chi \tilde{\chi})_{1}+\epsilon^{2}(\chi \tilde{\chi})_{2}+\mathcal{O}\left(\epsilon^{3}\right), \\
Y & =\frac{2 \epsilon}{\Lambda} m^{2}+\mathcal{O}\left(\epsilon^{3}\right),
\end{aligned}
$$


where $(\chi \tilde{\chi})_{1,2}$ denotes $\mathcal{O}\left(\epsilon^{1,2}\right)$ part of $\chi \tilde{\chi}$, we obtain

$$
\begin{aligned}
& (\chi \tilde{\chi})_{1}=0, \\
& (\chi \tilde{\chi})_{2}=\frac{m^{2}}{\Lambda^{2}}\left(2|\chi|^{2}-m^{2}\right) \approx \frac{m^{4}}{\Lambda^{2}} .
\end{aligned}
$$

The vacuum energy $V_{\text {local minimum }}$ at the local minimum (14) and $V_{\text {local maximum }}$ at the local maximum (15) can be calculated as

$$
\begin{aligned}
V_{\text {local minimum }} & =\left(N_{f}-N_{c}\right)\left|\left\langle\mathcal{O}\left(\epsilon^{2}\right)\right\rangle\right|^{2}+N_{c}\left|\tilde{m}^{2}\right|^{2}+\left(N_{f}-N_{c}\right) \frac{2 \epsilon^{2}}{\Lambda^{2}} m^{6}, \\
V_{\text {local maximum }} & =\left(N_{f}-N_{c}\right)\left|m^{2}\right|^{2}+N_{c}\left|\tilde{m}^{2}\right|^{2},
\end{aligned}
$$

which will be necessary to compute the lifetime of our metastable SUSY breaking vacuum.

\section{Calculation of Coleman-Weinberg potential for the pseudo-moduli $\Phi$}

At tree level, $\langle\Phi\rangle$ is undetermined since $\Phi$ does not appear in the tree level potential. Because of $F_{\Phi} \neq 0$, if the $\langle\Phi\rangle$ is lifted at the finite value by one-loop quantum corrections, SUSY breaking can be transmitted to the MSSM sector. To study the issue, we have to compute a Coleman-Weinberg potential for the moduli $\Phi$. All the massive fields at tree level contribute to the potential. Since we are interested in the moduli $\Phi$, we focus on the massive fields, $\rho, \tilde{\rho}, Z, \tilde{Z}$, whose masses depend on the $\operatorname{VEV}$ of $\Phi$.

The mass matrix for their bosonic components is calculated as

$$
\left(\rho^{\dagger}, \tilde{\rho}^{\dagger}, Z^{\dagger}, \tilde{Z}^{\dagger}\right) \mathcal{M}_{B}^{2}\left(\begin{array}{c}
\rho \\
\tilde{\rho} \\
Z \\
\tilde{Z}
\end{array}\right), \quad \mathcal{M}_{B}^{2} \equiv\left(\begin{array}{ll}
W^{\dagger i k} W_{k j} & W^{\dagger i j k} W_{k} \\
W_{i j k} W^{\dagger k} & W_{i k} W^{\dagger k j}
\end{array}\right),
$$

where

$$
\begin{aligned}
W^{\dagger i k} W_{k j} & \approx\left(\begin{array}{cccc}
m^{2}+|\Phi|^{2} & 4\left(\frac{\epsilon}{\Lambda}\right)^{2} m^{4} & m \Phi & 0 \\
4\left(\frac{\epsilon}{\Lambda}\right)^{2} m^{4} & m^{2}+|\Phi|^{2} & 0 & m \Phi \\
m \Phi^{\dagger} & 0 & m^{2} & 0 \\
0 & m \Phi^{\dagger} & 0 & m^{2}
\end{array}\right), \\
W^{\dagger i j k} W_{k} & \approx\left(\begin{array}{cccc}
2\left(\frac{\epsilon}{\Lambda}\right)^{2} m^{4} & \tilde{m}^{2} & 0 & m Y \\
\tilde{m}^{2} & 2\left(\frac{\epsilon}{\Lambda}\right)^{2} m^{4} & m Y & 0 \\
0 & m Y^{\dagger} & 0 & 0 \\
m Y^{\dagger} & 0 & 0 & 0
\end{array}\right)
\end{aligned}
$$


where $W_{a} \equiv \partial W / \partial \phi^{a}$ etc. The corresponding mass matrix for fermionic components $\mathcal{M}_{F}$ are obtained by taking the zero limit of SUSY breaking $\tilde{m}^{2}=0$. The parameter range without tachyonic messenger mass squareds is given as follows.

$$
-1+\frac{\tilde{m}^{2}}{m^{2}} \pm \mathcal{O}(1) \epsilon^{2}\left(\frac{m}{\Lambda}\right)^{2}<4 \epsilon \frac{m}{\Lambda} \frac{\Phi}{m}<+1+\frac{\tilde{m}^{2}}{m^{2}} \pm \mathcal{O}(1) \epsilon^{2}\left(\frac{m}{\Lambda}\right)^{2}
$$

We search for a local minimum with nonvanishing VEV of the pseudo moduli $\Phi$ in this range of parameters.

The Coleman-Weinberg potential is defined as

$$
V_{1-\text { loop }}=\frac{1}{64 \pi^{2}} \operatorname{Tr}\left[\mathcal{M}_{B}^{4} \ln \frac{\mathcal{M}_{B}^{2}}{\Lambda^{2}}-\mathcal{M}_{F}^{4} \ln \frac{\mathcal{M}_{F}^{2}}{\Lambda^{2}}\right]
$$

where $\Lambda$ is a cutoff scale. All massive fields except for a pseudo-moduli $\Phi$ contribute

to the Coleman-Weinberg potential. $Y, \chi, \tilde{\chi}$ have $\mathcal{O}(m)$ masses. $\rho, \tilde{\rho}, Z, \tilde{Z}$ have masses depending on the $\langle\Phi\rangle$ and the masses are turned out to be $m_{ \pm} \sim \mathcal{O}(m)$ or $\mathcal{O}(0.1 m)$ discussed later. We found that the minimum is shifted from the origin. One example is shown in Fig. 1 of section 6. In this numerical computation, we took all parameters to be real. As a consistency check, taking the limit $\epsilon=0, \tilde{m}^{2}=m^{2}$, we confirmed that the ISS results $\langle\Phi\rangle=0$ is reproduced.

\section{Mediation of Metastable SUSY breaking}

In the previous section, we found $\langle\Phi\rangle \neq 0$ at 1-loop, which implies that the gaugino mass can be generated if $\rho, \tilde{\rho}$ are identified with messengers and the standard model gauge group is embedded in the unbroken subgroup of the flavor symmetry $S U\left(N_{c}\right)$.

In our case, the mass matrix for the messengers takes of the form

$$
W \supset(\rho, Z)\left(\begin{array}{cc}
\Phi-2 \frac{\epsilon}{\Lambda} m^{2} & m \\
m & 0
\end{array}\right)\left(\begin{array}{c}
\tilde{\rho} \\
\tilde{Z}
\end{array}\right) \equiv(\rho, Z) \mathcal{M}\left(\begin{array}{c}
\tilde{\rho} \\
\tilde{Z}
\end{array}\right)
$$

The leading gaugino masses at the linear order of SUSY breaking $F_{\Phi}$ are given by the formula $m_{\lambda_{i}}=\frac{g_{i}^{2}}{(4 \pi)^{2}} N F_{\Phi} \frac{\partial}{\partial \Phi} \log \operatorname{det} \mathcal{M}$ where $N$ is a flavor number of messengers. Note that this leading gaugino masses vanish because $\operatorname{det} \mathcal{M}$ does not depend on $\Phi$ in (28).

Then, the gaugino masses in our case are generated at one-loop by the cubic order of SUSY breaking $\mathcal{O}\left(F_{\Phi}^{3}\right)$ [37]. On the other hand, the sfermion masses are generated in a usual gauge mediation form:

$$
\begin{aligned}
& m_{\lambda_{i}} \approx\left(N_{f}-N_{c}\right) \frac{\alpha_{i}}{4 \pi}\left(\frac{\left\langle F_{\Phi}\right\rangle}{\langle\Phi\rangle^{2}}\right)^{2} \frac{\left\langle F_{\Phi}\right\rangle}{\langle\Phi\rangle} \sim\left(N_{f}-N_{c}\right) \frac{\alpha_{i}}{4 \pi}\left(\frac{\tilde{m}}{m}\right)^{6} m \\
& m_{\tilde{f}}^{2} \approx\left(N_{f}-N_{c}\right) C_{i}\left(\frac{\alpha_{i}}{4 \pi}\right)^{2}\left(\frac{\left\langle F_{\Phi}\right\rangle}{\langle\Phi\rangle}\right)^{2} \sim\left(N_{f}-N_{c}\right) C_{i}\left(\frac{\alpha_{i}}{4 \pi}\right)^{2}\left(\frac{\tilde{m}}{m}\right)^{4} m^{2}
\end{aligned}
$$


where $\alpha_{i}$ are fine structure constants for the standard model gauge group $i=S U(3)_{C}$, $S U(2)_{L}, U(1)_{Y} \cdot C_{i}$ are the corresponding quadratic Casimir coefficients. We can see that the sfermion masses are heavier than the gaugino masses,

$$
\frac{m_{\tilde{f}}}{m_{\lambda}} \sim \sqrt{\frac{C_{i}}{N_{f}-N_{c}}}\left(\frac{m}{\tilde{m}}\right)^{4} .
$$

To avoid a huge hierarchy between the gaugino masses and the sfermion ones, the ratio $\tilde{m}^{2} / m^{2}$ must be at most 0.1 . Otherwise, the large one-loop corrections to Higgs mass by the third generation squarks require unnatural tuning of parameters to solve the gauge hierarchy problem. To obtain $m_{\lambda} \sim \mathcal{O}(100 \mathrm{GeV})$, we need

$$
\left(\frac{\tilde{m}}{m}\right)^{6} m \sim 10 \mathrm{TeV}
$$

For instance, the sfermion masses become $10 \mathrm{TeV}$ for $\tilde{m}^{2} / m^{2} \sim 0.1$.

The gravitino mass, which is the lightest superparticle in the gauge mediation, is estimated as

$$
m_{3 / 2} \simeq \frac{F_{\Phi}}{\sqrt{3} M_{P}} \sim 10^{-11}\left(\frac{m}{\tilde{m}}\right)^{10} \mathrm{GeV}
$$

where $M_{P}$ is a reduced Planck scale $2.4 \times 10^{18} \mathrm{GeV}$. For instance, the gravitino mass becomes $m_{3 / 2} \sim 1 \mathrm{keV}$ for $\tilde{m}^{2} / m^{2} \sim 0.1$, which avoids the gravitino problem 38.

\section{The lifetime of the Metastable vacuum}

We have to check whether our SUSY breaking vacuum is long-lived compared to the age of universe since our SUSY breaking vacuum is not a global minimum of the potential but a local one. To estimate the decay rate from our false vacuum to the true SUSY vacuum, we need the VEV of a SUSY vacuum.

Let us consider the case $\langle M\rangle \neq 0$ where the dual quarks $q, \tilde{q}$ are decoupled in the low energy effective theory below the scale $\langle M\rangle$. In other words, the low energy effective theory is an $S U\left(N_{f}-N_{c}\right)$ super Yang-Mills theory.

SUSY conditions for $q, \tilde{q}$ are

$$
\begin{aligned}
& 0=\frac{\partial W}{\partial q}=\left(M+2 \frac{\epsilon}{\Lambda} q \tilde{q}\right) \tilde{q}, \\
& 0=\frac{\partial W}{\partial \tilde{q}}=q\left(M+2 \frac{\epsilon}{\Lambda} q \tilde{q}\right) .
\end{aligned}
$$

We find two vacua.

$$
q=\tilde{q}=0(\operatorname{vacuum}(\mathrm{i})), \quad M+2 \frac{\epsilon}{\Lambda} q \tilde{q}=0(\operatorname{vacuum}(\mathrm{ii})) .
$$


In the vacuum (i) case, the effective superpotential becomes

$$
W_{\text {eff }}=m^{2} \operatorname{Tr} M+\left(N_{f}-N_{c}\right) \Lambda_{L}^{3}=m^{2} \operatorname{Tr} M+\left(N_{f}-N_{c}\right)\left[\Lambda^{3\left(N_{f}-N_{c}\right)-N_{f}} \operatorname{det} M\right]^{\frac{1}{N_{f}-N_{c}}}
$$

where $\Lambda_{L}$ is a dynamical scale of $S U\left(N_{f}-N_{c}\right)$ super Yang-Mills theory and the second term is generated by the gaugino condensation of super Yang-Mills theory. 1-loop matching condition for the holomorphic gauge coupling at the scale $\langle M\rangle$,

$$
\Lambda_{L}^{3\left(N_{f}-N_{c}\right)}=\Lambda^{3\left(N_{f}-N_{c}\right)-N_{f}} \operatorname{det} M
$$

is used in the second equality. SUSY condition for $M$

$$
0=\frac{\partial W_{\mathrm{eff}}}{\partial M}=m^{2}+\Lambda^{\frac{3\left(N_{f}-N_{c}\right)-N_{f}}{N_{f}-N_{c}}}(\operatorname{det} M)^{\frac{1}{N_{f}-N_{c}}} / M
$$

tells us that the SUSY vacuum is located at

$$
\langle M\rangle \sim\left(\frac{m}{\Lambda}\right)^{\frac{2\left(N_{f}-N_{c}\right)}{N_{c}}} \Lambda .
$$

In the vacuum (ii) case, the effective superpotential takes the form

$$
\begin{aligned}
W_{\mathrm{eff}} & =-\frac{\Lambda}{4 \epsilon} \operatorname{Tr} M^{2}+m^{2} \operatorname{Tr} M+\left(N_{f}-N_{c}\right) \Lambda_{L}^{3} \\
& =-\frac{\Lambda}{4 \epsilon} \operatorname{Tr} M^{2}+m^{2} \operatorname{Tr} M+\left(N_{f}-N_{c}\right)\left[\Lambda^{3\left(N_{f}-N_{c}\right)-N_{f}} \operatorname{det} M\right]^{\frac{1}{N_{f}-N_{c}}}
\end{aligned}
$$

SUSY condition for $M$

$$
0=\frac{\partial W_{\mathrm{eff}}}{\partial M}=-\frac{\Lambda}{2 \epsilon} M+m^{2}+\Lambda^{\frac{3\left(N_{f}-N_{c}\right)-N_{f}}{N_{f}-N_{c}}} M^{\frac{N_{c}}{N_{f}-N_{c}}}
$$

provides

$$
\langle M\rangle \sim\left(\frac{1}{2 \epsilon}\right)^{\frac{N_{f}-N_{c}}{2 N_{c}-N_{f}}} \Lambda
$$

where $m^{2}$ in the SUSY condition is neglected.

We are now in a position to estimate the decay rate from the false vacuum to the true vacuum. The necessary ingredients to estimate are the potential height $V_{\text {peak }}$ and the distance between the false vacuum and the true one in the field space $\Delta \Phi$. In our case, the potential height at the corresponding local maximum

$$
\langle\Phi\rangle \simeq m, \quad\langle Y\rangle=\frac{2 \epsilon}{\Lambda} m^{2}, \quad|\chi|=|\tilde{\chi}|=0
$$

is

$$
V_{\text {peak }}=\left(N_{f}-N_{c}\right)\left|m^{2}\right|^{2}+N_{c}\left|\tilde{m}^{2}\right|^{2} \approx\left|m^{2}\right|^{2}
$$


The field distance between two vacua is

$$
\Delta \Phi \approx\langle M\rangle_{\text {SUSY vacuum }}=\left\{\begin{array}{l}
\left(\frac{m}{\Lambda}\right)^{\frac{2\left(N_{f}-N_{c}\right)}{N_{c}}} \Lambda(\operatorname{vacuum}(\mathrm{i})) \\
\left(\frac{1}{2 \epsilon}\right)^{\frac{N_{f}-N_{c}}{2 N_{c}-N_{f}}} \Lambda(\operatorname{vacuum}(\mathrm{ii}))
\end{array}\right.
$$

Then, the bounce action is estimated as

$$
S \sim \frac{(\Delta \Phi)^{4}}{V_{\text {peak }}} \sim\left\{\begin{array}{c}
\left(\frac{\Lambda}{m}\right)^{4-8\left(N_{f}-N_{c}\right) / N_{c}} \gg 1(\operatorname{vacuum}(\mathrm{i})), \\
\left(\frac{1}{2 \epsilon}\right)^{4\left(N_{f}-N_{c}\right) /\left(N_{c}-\left(N_{f}-N_{c}\right)\right)}\left(\frac{\Lambda}{m}\right)^{4} \gg 1(\operatorname{vacuum}(\mathrm{ii})) .
\end{array}\right.
$$

The bounce action can be parametrically large as long as we take $m / \Lambda \ll 1$.

For instance, let us consider the case $m / \Lambda=0.1, \epsilon=1, N_{f}=6$ and $N_{c}=5$ which gives the most stringent bound, then we obtain

$$
S>10^{2.4}(\operatorname{vacuum}(\mathrm{i})), \quad 4 \times 10^{3}(\operatorname{vacuum}(\mathrm{ii})) .
$$

The lifetime of the metastable vacuum can be obtained

$$
\tau \sim e^{S}>e^{251}(\operatorname{vacuum}(\mathrm{i})), \quad e^{4000}(\operatorname{vacuum}(\mathrm{ii}))
$$

which implies that our SUSY breaking vacuum is sufficiently long-lived compared to the age of the universe $\left(\sim e^{40}\right)$.

\section{Landau pole analysis}

In this section, we examine whether the QCD coupling constant at the GUT scale is perturbative or not when the standard model gauge group is embedded in the unbroken subgroup of the flavor symmetry $S U\left(N_{c}\right)$.

One-loop gauge coupling RGE is given by

$$
g_{i}^{-2}(\mu)=g_{i}^{-2}\left(\mu^{\prime}\right)+\frac{b_{i}}{8 \pi^{2}} \ln \left(\frac{\mu}{\mu^{\prime}}\right)
$$

where $b_{i}$ is one-loop beta function coefficient of the gauge group $i=S U(3)_{c}, S U(2)_{L}, U(1)_{Y}$.

The one-loop beta function coefficients for QCD coupling at various scales are listed below.

$$
\begin{aligned}
\mu<m_{\lambda} & : \quad b_{3}=b_{3}^{\mathrm{SM}}=7 \\
m_{\lambda}<\mu<m_{\tilde{f}} \sim(m / \tilde{m})^{4} m_{\lambda} & : \quad b_{3}=b_{3}^{\mathrm{SM}}-\frac{2}{3} \times 3=5 \\
m_{\tilde{f}} \sim(m / \tilde{m})^{4} m_{\lambda}<\mu<m_{-} & : \quad b_{3}=b^{\mathrm{MSSM}}=3 \\
m_{-}<\mu<m_{+} & : \quad b_{3}=b_{3}^{\mathrm{MSSM}}-b_{3}^{\Phi}-\left(N_{f}-N_{c}\right)=-\left(N_{f}-N_{c}\right) \\
m_{+}<\mu<\Lambda & : \quad b_{3}=-2\left(N_{f}-N_{c}\right)
\end{aligned}
$$


where $b^{\mathrm{SM}}, b^{\mathrm{MSSM}}$ are the QCD one-loop beta function coefficients for the standard model and the minimal SUSY standard model. \pm of $m_{ \pm}$means that the sign of the square root in the mass eigenvalues for messengers. Numerically, it is roughly $m_{+} \sim m, m_{-} \sim 0.1 m$. $\Phi$ is an adjoint representation under $S U\left(N_{c}\right)$, which gives $b_{3}^{\Phi}=3$ for QCD color gauge group $S U(3)_{c}$. Here we use the numerically obtained mass $m_{\Phi} \sim 0.1 \mathrm{~m}$.

Taking the following values

$$
\Lambda=M_{\mathrm{GUT}} \sim 10^{16} \mathrm{GeV}, \quad \frac{g_{3}^{2}\left(M_{Z}\right)}{4 \pi} \sim 0.18,
$$

and one-loop beta function coefficients obtained above, the QCD coupling is expressed as

$$
\begin{aligned}
\alpha_{3}\left(M_{\mathrm{GUT}}\right)^{-1} \sim & 8.5-\frac{1}{2 \pi}\left[-0.74+\left\{13+6\left(N_{f}-N_{c}\right)\right\} \ln \left(\frac{\tilde{m}}{m}\right)^{2}\right. \\
& \left.+2.3\left\{25\left(N_{f}-N_{c}\right)-3\right\}\right] .
\end{aligned}
$$

Requiring that the QCD coupling constant is perturbative at the GUT scale, the constraint for the flavor number of messengers $N_{f}-N_{c}$ and $\tilde{m}^{2} / m^{2}$ can be found,

$$
\alpha_{3}\left(M_{\mathrm{GUT}}\right)<1 \Leftrightarrow\left[13+6\left(N_{f}-N_{c}\right)\right] \ln \left(\frac{\tilde{m}}{m}\right)^{2}+57.5\left(N_{f}-N_{c}\right)<61.1 .
$$

The examples satisfying (54) are $N_{f}-N_{c} \leq 2$ for $(\tilde{m} / m)^{2}=0.1$ and $N_{f}-N_{c} \leq 4$ for $(\tilde{m} / m)^{2}=0.01$. The smaller $(\tilde{m} / m)^{2}$ allows a larger number of messengers $N_{f}-N_{c}$, but the hierarchy between the gaugino masses and the sfermion ones becomes larger. Thus,

$$
N_{f}-N_{c}=1,2
$$

are phenomenologically favorable. It is important to mention the differences between our model and the other relevant models that Landau pole problem is avoided when the embedding of the standard model gauge group in $S U\left(N_{f}-N_{c}\right)$ not in $S U\left(N_{c}\right)$ in the model [21] and cannot be avoided in the model [34]. Taking into account the facts that the free magnetic description is valid for $N_{c}+1 \leq N_{f}<\frac{3}{2} N_{c}$ and $S U\left(N_{c}\right)$ embedding of the standard model gauge group is possible only for $N_{c} \geq 5$, the allowed ranges of the number of colors and flavors are found

$$
5 \leq N_{c}, \quad 6 \leq N_{f}<\frac{3}{2} N_{c}
$$

A numerical result for a metastable SUSY breaking minimum found in this analysis 
is summarized in the table and Fig. 11 below.

\begin{tabular}{|c|c|c|}
\hline$\langle\Phi\rangle / m$ & -1.18 & -1.18 \\
\hline$\tilde{m}^{2} / m^{2}$ & 0.1 & 0.1 \\
\hline$\epsilon$ & 1 & 1 \\
\hline$m / \Lambda$ & 0.1 & 0.1 \\
\hline$m_{\lambda}(\mathrm{GeV})$ & 100 & 100 \\
\hline$m_{\tilde{f}}(\mathrm{TeV})$ & 10 & 10 \\
\hline lifetime & $\gg \tau_{0}$ & $>\tau_{0}\left(N_{c} \geq 8\right)$ \\
\hline$N_{f}-N_{c}$ & 1 & 2 \\
\hline
\end{tabular}

Here $\tau_{0}$ means the age of universe. The constraint of color number in $N_{f}-N_{c}=2$ case

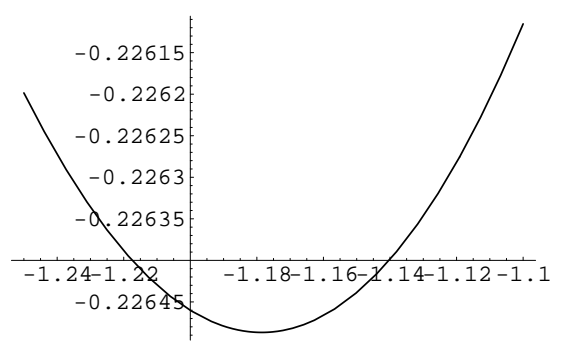

Figure 1: One-loop Coleman-Weinberg potential for the pseudo moduli $\Phi$. The vertical axis denotes $V_{1-\text { loop }} / m^{4}$, and the horizontal axis does $\Phi / m$.

comes from the lifetime of our metastable SUSY breaking vacuum (decay to the vacuum (i)) and the allowed region from Landau pole analysis,

$$
\left(\frac{\Lambda}{m}\right)^{4-8\left(N_{f}-N_{c}\right) / N_{c}}>40 .
$$

No constraint for the number of color arises from the decay to the vacuum (ii).

\section{Summary}

We have constructed a model of direct gauge mediation of metastable SUSY breaking by simply deforming the ISS model in terms of a dual meson superpotential mass term, which breaks an $U(1)_{R}$ explicitly. No extra matter field is introduced. It was shown that the VEV of the pseudo moduli $\Phi$ is shifted at the finite value through the Coleman-Weinberg potential for the pseudo moduli. The lifetime of our metastable vacuum was turned out to be sufficiently long compared to the age of universe as long as $m / \Lambda \ll 1$. By gauging a subgroup of the flavor symmetry $S U\left(N_{c}\right)$, the direct gauge mediation was realized. In our model, the sfermion masses are heavier than the gaugino masses. Typically, sfermion masses are $10 \mathrm{TeV}$ corresponding to the SUSY breaking scale of order $10^{6} \mathrm{GeV}$. The condition for the QCD coupling to be perturbative at the GUT scale was derived and it 
is found that Landau pole problem can be avoided. From these analysis, the parameters in the SUSY breaking sector have been phenomenologically constrained.

It is deserved to pay an attention that our simple deformed ISS model provides a phenomenologically viable model of the direct gauge mediation of metastable SUSY breaking. Introducing only the term $\operatorname{Tr}(q \tilde{q})^{2}$ among the same order other operators is just an assumption, but might be explained from the view point of brane picture. We can say at least that SUSY is not restored even if more general higher dimensional operators $\operatorname{Tr}(q \tilde{q})^{n}(n$ : integer more than 2$)$ are added to the superpotential since SUSY breaking condition $\partial W / \partial \Phi \neq 0$ is unchanged. This tells us that our conslusion obtained in this paper is essentially unchanged. Adding the operators including $M$ to the superpotential would spoil our conslusion since SUSY is restored. We hope that our model discussed in this paper will shed some insights for further studies on the model building of metastable SUSY breaking.

\section{Acknowledgments}

We thank C. Csaki, C. Durnford, Y. Hyakutake, J. Jaeckel, T. Kawano and R. Kitano for useful discussions. The work of the authors was supported in part by the Grant-in-Aid for Scientific Research of the Ministry of Education, Science and Culture, No.16540258 (N.H.), No.17740146 (N.H.) and No.18204024 (N.M.).

\section{References}

[1] E. Witten, Nucl. Phys. B 188, 513 (1981).

[2] E. Witten, Nucl. Phys. B 202, 253 (1982).

[3] K. I. Izawa and T. Yanagida, Prog. Theor. Phys. 95, 829 (1996).

[4] K. A. Intriligator and S. D. Thomas, Nucl. Phys. B 473, 121 (1996).

[5] M. Dine and W. Fischler, Phys. Lett. B 110, 227 (1982); L. Alvarez-Gaume, M. Claudson and M. B. Wise, Nucl. Phys. B 207, 96 (1982); S. Dimopoulos and S. Raby, Nucl. Phys. B 219, 479 (1983).

[6] M. Dine, A. E. Nelson and Y. Shirman, Phys. Rev. D 51, 1362 (1995); M. Dine, A. E. Nelson, Y. Nir and Y. Shirman, Phys. Rev. D 53, 2658 (1996).

[7] I. Affleck, M. Dine and N. Seiberg, Nucl. Phys. B 256, 557 (1985).

[8] E. Poppitz and S. P. Trivedi, Phys. Rev. D 55, 5508 (1997). 
[9] N. Arkani-Hamed, J. March-Russell and H. Murayama, Nucl. Phys. B 509, 3 (1998).

[10] H. Murayama, Phys. Rev. Lett. 79, 18 (1997).

[11] S. Dimopoulos, G. R. Dvali, R. Rattazzi and G. F. Giudice, Nucl. Phys. B 510, 12 (1998).

[12] N. Okada, Phys. Lett. B 423, 305 (1998).

[13] K. Agashe, Phys. Lett. B 435, 83 (1998).

[14] N. Maru, Phys. Lett. B 436, 311 (1998).

[15] K. Intriligator, N. Seiberg and D. Shih, JHEP 0604, 021 (2006).

[16] N. Seiberg, Nucl. Phys. B 435, 129 (1995).

[17] S. Forste, Phys. Lett. B 642, 142 (2006).

[18] A. Amariti, L. Girardello and A. Mariotti, JHEP 0612, 058 (2006).

[19] M. Dine, J. L. Feng and E. Silverstein, Phys. Rev. D 74, 095012 (2006).

[20] M. Dine and J. Mason, arXiv:hep-ph/0611312.

[21] R. Kitano, H. Ooguri and Y. Ookouchi, Phys. Rev. D 75, 045022 (2007).

[22] H. Murayama and Y. Nomura, Phys. Rev. Lett. 98, 151803 (2007); Phys. Rev. D 75, 095011 (2007).

[23] O. Aharony and N. Seiberg, JHEP 0702, 054 (2007).

[24] C. Csaki, Y. Shirman and J. Terning, JHEP 0705, 099 (2007).

[25] S. A. Abel and V. V. Khoze, arXiv:hep-ph/0701069.

[26] S. A. Abel, J. Jaeckel and V. V. Khoze, arXiv:hep-ph/0703086.

[27] D. Shih, arXiv:hep-th/0703196.

[28] K. Intriligator, N. Seiberg and D. Shih, JHEP 0707, 017 (2007).

[29] L. Ferretti, arXiv:0705.1959 [hep-th].

[30] A. Katz, Y. Shadmi and T. Volansky, JHEP 0707, 020 (2007).

[31] F. Brummer, JHEP 0707, 043 (2007). 
[32] Y. Bai, J. Fan and Z. Han, Phys. Rev. D 76, 065003 (2007).

[33] H. Y. Cho and J. C. Park, arXiv:0707.0716 [hep-ph].

[34] S. Abel, C. Durnford, J. Jaeckel and V. V. Khoze, arXiv:0707.2958 [hep-ph].

[35] M. Arai, C. Montonen, N. Okada and S. Sasaki, arXiv:0708.0668 [hep-th].

[36] H. Abe, T. Kobayashi and Y. Omura, arXiv:0708.3148 [hep-th].

[37] K. I. Izawa, Y. Nomura, K. Tobe and T. Yanagida, Phys. Rev. D 56, 2886 (1997).

[38] T. Moroi, H. Murayama and M. Yamaguchi, Phys. Lett. B 303, 289 (1993). 